\title{
Preferential processing of target features in texture segmentation
}

\author{
CHARLES T. SCIALFA and KENNETH M. JOFFE \\ University of Calgary, Calgary, Alberta, Canada
}

\begin{abstract}
In five experiments, observers were required to detect a texture target and/or identify the orientation of elements composing target and nontarget regions. They were significantly worse at discerning the orientation of nontarget regions than at detecting target presence (Experiment 1). On the other hand, accuracy of identifying target orientation was found to be near 100\% (Experiment 2). When observers were required only to identify surround orientation (Experiment 3), accuracy was diminished on target-present trials relative to that on target-absent trials. The superiority of target processing and the interference produced by target presence on surround processing were demonstrated in unpracticed observers (Experiment 4). In Experiment 5, it was found that information regarding target presence is available before information regarding feature values of the target. These findings are consistent with a model of visual attention and search that incorporates a fast generalized difference operator and a slower feature comparison process.
\end{abstract}

In a typical texture segmentation task, a small region of uniform orientation is embedded in a surrounding region of a different orientation. The texture target (i.e., figure, texture patch) is rapidly detected if the featural gradient between the two regions is sufficiently large. It is often asserted that segmentation is based on the detection of a generalized difference between the two regions of the display. A goal of the research reported here was to determine if, in addition to detecting a difference, observers could report the identity of the features composing the texture target and nontarget regions. A second and related goal was to determine if the time course of the generalized difference operator (GDO) differs from that of the processes providing specific feature values.

Nothdurft (1985b, 1991 a, 1991b, 1992), Julesz (1986), Sagi and Julesz (1987), and Wolfe (1992) have emphasized the importance to texture segmentation and feature search of a nonspecific differencing operator that is sensitive to a texture gradient (e.g., a local orientation difference) but not to specific features. Such a mechanism is central to computational models of texture segmentation (Landy \& Bergen, 1991; Malik \& Perona, 1990; Voorhees \& Poggio, 1988), as well as the bottom-up component of the original and recently revised Guided Search Model (Cave \& Wolfe, 1990; Wolfe, 1994). A differencing operator was invoked by Atkinson and Braddick (1989) to account for the finding that target local-

Support for this research was provided, in part, by funding from the Natural Science and Engineering Research Council of Canada. The authors would like to thank Don Kline, David Thomas, Jeremy Wolfe, Lester Krueger, and two anonymous reviewers for valuable comments on earlier versions of this manuscript. Correspondence should be addressed to Charles T. Scialfa, Department of Psychology, University of Calgary, Calgary, AB, Canada T2N 1N4 (e-mail: scialfa@acs. ucalgary.ca). ization in feature search occurs more rapidly than does target identification. They argued that coarse localization is based on the detection of "feature gradients" that can "signal where without knowing what the target is, except that it is different"'(Atkinson \& Braddick, 1989, p. 182). Sagi and Julesz (1985a, 1985b) also found that localization and detection of multiple feature targets occurred more quickly than did discrimination among the targets. It seems, then, that specific feature values (i.e., "what") become available only after a featurally undifferentiated object has been detected and crudely localized.

One of the possible functions of a GDO is to direct attention. A fast GDO operating in a bottom-up fashion signals unique regions of a scene. A categorical representation of the GDO is embodied in the bottom-up component of Guided Search 2.0 (GS2; see Wolfe, 1994). A GDO would also respond to singletons (Hillstrom \& Yantis, 1994; Jonides \& Yantis, 1988; Theeuwes, 1991, 1992) indicating the presence of a novel object (Hillstrom \& Yantis, 1994).

Texture segmentation and feature search tasks can involve not only the detection of a feature difference but also the identification of specific feature values. The latter process is often conceived as a dimension-bydimension comparison of representations of display elements and sought objects.

According to GS2 (Wolfe, 1994), both processes provide input to an activation map. Specific featural information is lost in the summation; only relative activation is available. Activation that results from the GDO will, in the case of a texture patch, produce a more-or-less hollow outline of the patch. Activation resulting from the feature comparison process will produce greatest activation for the entire target region.

The continuously updated activation map is used to guide visual attention to the regions that are most likely 
to contain the sought object. It seems likely that attentional allocation proceeds in parallel with the construction of the activation map. In consequence, though not stated explicitly in GS2, the output of a faster GDO can guide visual attention before a slower feature comparison process has contributed to the activation map.

What are the implications of this view for texture segmentation tasks? If the border outline of a unique region is made available prior to specific featural information, then attention may be directed to that region regardless of the features contained within it. Attention is likely directed to the spatial average of the boundary coordinates--that is, to the center of the unique region, as demonstrated in several "center-of-gravity" studies (Findlay, 1982; Ottes, Van Gisbergen, \& Eggermont, 1984, 1985).

As attention is guided to a unique region, specific featural information pertaining to it may rapidly become available. Conversely, information contained within nontarget regions can only be obtained later, after attention is shifted away from the unique region. If processing is limited to a very brief duration, there may not be sufficient time for the attention shift to occur, and surround orientation identification will suffer. Experiment 1 was designed to test this prediction.

\section{EXPERIMENT 1 Target Detection and Identification of Surround Orientation}

In Experiment 1, observers were required to detect a brief, backward-masked texture target composed of uniformly oriented lines embedded in a uniformly oriented surround. In addition, they were required to indicate the orientation of the surround. Detection should be relatively simple. However, if detection is based on a GDO that directs attention to the unique region, one may expect that identifying the feature values in the surrounding region would be difficult, particularly at the brief exposure durations employed.

\section{Method}

Observers. Five observers $(M=29.4$ years of age, range $=$ 24-38 years) participated. All were from the Department of Psychology at the University of Calgary. They had normal or corrected visual acuity of 20/22 or better and were experienced with laboratory visual search tasks.

Materials and Apparatus. All displays were created and data collected using VScope version 1.2b (Rensink \& Enns, 1993). Displays were presented on a Macintosh IIx computer with a $53-\mathrm{cm}$ high-resolution color monitor set to black and white. Oral responses were recorded manually.

A height-adjustable chinrest and chair were used to stabilize head position and to ensure a gaze angle of approximately $0^{\circ}$. Trial onset was observer-initiated with a buttonpress on a standard keyboard situated directly in front of the observer. Luminance was measured using a Minolta (LS-100) luminance meter.

Stimuli. The fixation stimulus was composed of five black dots, which had a luminance of $0.311 \mathrm{~cd} / \mathrm{m}^{2}$. Four of these dots were $0.255^{\circ}$ in diameter and were placed at and within the four corners of an imaginary square subtending $1.02^{\circ} \times 1.02^{\circ}$. The remaining dot, centered within the imaginary square, was $0.382^{\circ}$ in diameter.
With a screen luminance of $80.09 \mathrm{~cd} / \mathrm{m}^{2}$, the Michelson contrast $\left(\mathrm{L}_{\max }-\mathrm{L}_{\min } / \mathrm{L}_{\max }+\mathrm{L}_{\min }\right)$ of the stimulus was $99 \%$.

Texture displays, subtending $20.16^{\circ} \times 20.16^{\circ}$, were composed of 336 black lines, each $0.764^{\circ}$ in length and $0.127^{\circ}$ in width. The minimum and maximum line separation was $0.127^{\circ}$ and $0.637^{\circ}$, respectively. Lines had the same luminance and contrast as the fixation stimulus. All lines were irregularly placed to control for any luminance cues that could facilitate target detection (Nothdurft, 1985a). Space-averaged luminance of texture displays was $77.40 \mathrm{~cd} / \mathrm{m}^{2}$.

Target-absent displays were composed of lines uniformly oriented at either $45^{\circ}$ to the left or right of vertical. Target-present displays, as shown in Figure 1a, were created by replacing nine oblique lines with a $3 \times 3$ matrix of vertical lines arranged within an imaginary square subtending $2.16^{\circ} \times 2.16^{\circ}$. The target square was positioned randomly at one of five locations corresponding to fixation or $2.29^{\circ}$ left, right, above, or below fixation along the cardinal meridia.

Masking displays, as shown in Figure $1 \mathrm{~b}$, were composed of stimuli with three bisecting lines oriented at $90^{\circ}$ (vertical) and $\pm 45^{\circ}$. Masks were created by copying the texture displays and adding the two necessary line segments to them. This served to mask any of the three possible line segments present in the previous texture display.

Procedure. Displays were viewed binocularly from a distance of $45 \mathrm{~cm}$. The observers were instructed to maintain fixation at all times. They were told that, because target location was randomized, no advantage would be gained by shifting gaze away from fixation.

Each trial began with the presentation of the fixation stimulus for $749 \mathrm{msec}$. A blank interval of $203 \mathrm{msec}$ was followed by the presentation of the texture display for $70 \mathrm{msec}$. The mask was presented for $1,400 \mathrm{msec}$ immediately after the offset of the texture display.

A two-stage verbal response was required for each trial. The observers were instructed to first determine whether the target was present or not and to subsequently identify surround orientation.

After 10 practice trials in which verbal feedback was provided, each observer completed 280 trials, separated into seven blocks of 40 trials each. There were 141 target-absent and 139 target-present trials. Both surround orientations occurred with equal frequency. Feedback was not provided during the testing phase.

Following participation in Experiments $l$ and 2 (below), the observers were retested in Experiment 1 to obtain information related to the effects of practice.

\section{Results}

Table 1 depicts averaged accuracy for both initial testing and retesting. In the initial testing phase, target detection $(83.34 \%)$ was significantly better than surround orientation accuracy $(45.74 \%)[t(4)=6.64, p<.01]$. In fact, the accuracy of surround orientation was no better or worse than would be expected on the basis of chance.

We were also interested in whether the accuracy of surround orientation was related to target presence. Table 1 includes surround orientation accuracy separately for target-present and target-absent trials. Although the observers were better at identifying surround orientation when the target was absent $(47.90 \%$ correct) as opposed to present $(43.46 \%$ correct), the difference was nonsignificant $[t(4)=2.13, p>.05]$.

It is evident that retest performance was better than performance in the initial testing phase, with the greatest improvement shown in the ability to discern the orientation of the surround. However, target identification accuracy $(95.00 \%)$ was still significantly better than the 
a

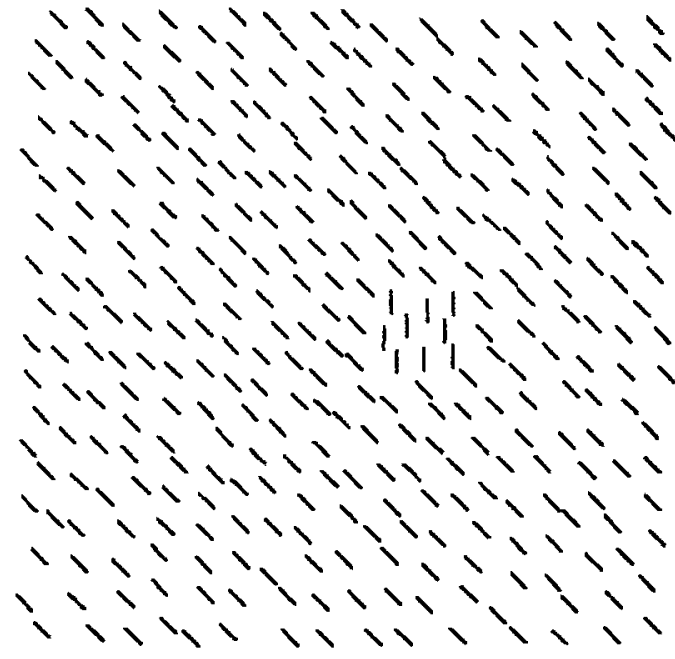

b

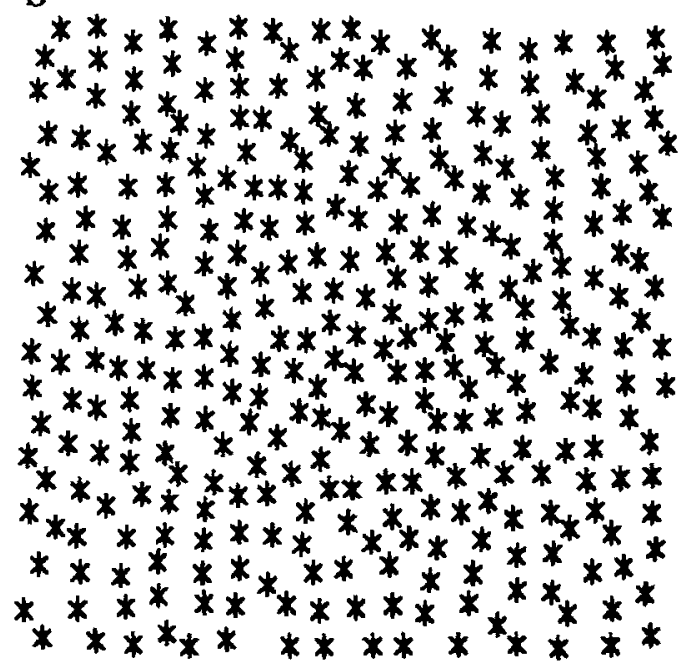

C

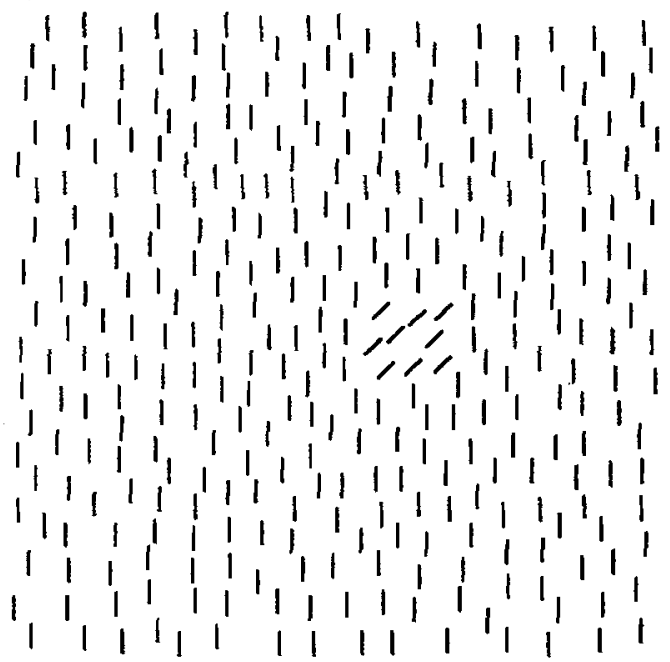

Figure 1. (a) Scaled example of the texture displays from Experiment 1 . The target region is the $3 \times 3$ matrix of vertically oriented lines. In this case, the correct response is "target-present," and the surround items are oriented "to the left." (b) Scaled example of the masking displays used in Experiment 1. (c) Scaled example of the texture displays from Experiment 2, where surround orientation was always vertical and target orientation was $45^{\circ}$ left or right of vertical.

accuracy found for surround orientation $(80.74 \%)$ $[t(4)=5.72, p<.01]$. In addition, and in contrast to that in the initial testing phase, surround orientation accuracy in the retesting phase was significantly better on targetabsent trials $(87.20 \%)$ than on target-present trials $(74.08 \%)[t(4)=3.73, p<.05]$.

\section{Discussion}

The results of the initial testing phase of Experiment 1 indicate that surround orientation accuracy was at levels no better than chance, even though target detection accuracy exceeded $80 \%$. This finding is consistent with the view that target detection resulted from a fast GDO and that the shift of attention to the surround could not be completed before mask onset. Alternatively, poorer sur- round identification may have resulted because instructions directed attention first to the target and only later to the surround. Either view is consistent with the retesting data, wherein surround orientation accuracy was significantly better on target-absent trials than on targetpresent trials.

\section{EXPERIMENT 2}

\section{Target Detection and Orientation Identification}

If attention is directed to the target region, either by instruction or the output of the GDO, then identification of target features should be easy, relative to surround identification. In Experiment 2, therefore, we omitted the demand to report surround orientation, asking observers to 
Table 1

Averaged Accuracy (in Percentage) and Standard Errors for Experiments 1-4

\begin{tabular}{|c|c|c|c|c|c|c|c|c|c|c|}
\hline \multirow[b]{3}{*}{ Task } & \multicolumn{4}{|c|}{ Experiment 1} & & & & & & \\
\hline & \multicolumn{2}{|c|}{ Initial Testing } & \multicolumn{2}{|c|}{ Retesting } & \multicolumn{2}{|c|}{ Experiment 2} & \multicolumn{2}{|c|}{ Experiment 3} & \multicolumn{2}{|c|}{ Experiment 4} \\
\hline & $\%$ & $S E$ & $\%$ & $S E$ & $\%$ & $S E$ & $\%$ & $S E$ & $\%$ & $S E$ \\
\hline Target detection & 83.34 & 2.54 & 95.00 & 1.52 & 98.36 & 0.24 & & & 95.55 & 2.01 \\
\hline Target identification (TP) & & & & & 96.70 & 1.41 & & & 92.98 & 3.63 \\
\hline Surround identification & 45.74 & 6.91 & 80.74 & 2.38 & & & 93.44 & 2.34 & 84.28 & 3.23 \\
\hline Surround identification (TP) & 43.46 & 7.45 & 74.08 & 3.43 & & & 89.96 & 3.46 & 75.55 & 4.21 \\
\hline Surround identification (TA) & 47.90 & 6.52 & 87.20 & 2.40 & & & 96.88 & 1.47 & 92.93 & 2.59 \\
\hline
\end{tabular}

Note-TP, target-present trials; TA, target-absent trials.

detect and, on target-present trials, to report the orientation of a texture target.

\section{Method}

Except for the following alterations, all observers, materials, and stimuli, as well as the general procedure, were identical to those of Experiment 1. Target-absent displays were composed of 336 vertically oriented lines. Target-present displays were composed of 327 vertically oriented lines and a target square of nine lines oriented $45^{\circ}$ to the left or right of vertical. Of the targetpresent trials, there were equal numbers of "left" $(n=70)$ and "right" $(n=69)$ displays. An example is shown in Figure 1c.

Oral responses were conditional on target presence. The observers were instructed to give an "absent" response if the target was not detected. They were instructed to indicate the orientation of target lines if the target was present.

\section{Results}

Observers were very accurate at detecting the target. As indicated in Table 1, accuracy of detection, collapsed across target-present and target-absent trials, averaged $98.36 \%$. They were also very accurate at identifying target orientation on target-present trials, averaging $96.70 \%$. The difference between detection and identification accuracy was nonsignificant $[t(4)=1.18, p=.304]$.

We also compared the identification accuracy of surround orientation on target-present and target-absent displays in the retesting phase of Experiment 1 with the identification accuracy of target orientation in the present experiment. Target identification accuracy was significantly better than surround accuracy on both targetpresent trials $[t(4)=6.44, p<.01]$ and target-absent trials $[t(4)=8.13, p<.01]$.

\section{Discussion}

The results of Experiment 2 indicate that the observers were as accurate at determining target orientation as they were at detecting target presence. Thus, the poor surround orientation accuracy in Experiment 1 could not have been due to the fact that the exposure duration was too brief to allow attention to be directed to any display region. Of greater interest, target orientation accuracy was better than surround orientation accuracy (Experiment 1, retesting phase). The superiority of target processing could not have been the result of greater practice, since the retesting phase of Experiment 1 followed the completion of Experiment 2.
The target superiority effect may have resulted because instructions directed the observers first to the target location. Recall that, in Experiment 1, surround orientation was reported only after judgments and responses concerning target presence. Attention must be allocated to at least two display locations. Duncan (1984, 1993 ) has shown that this is particularly difficult. In contrast, in Experiment 2, both presence and orientation information occurred at the same location, so that no spatial reallocation of attention was needed.

Alternatively, even in the absence of instructions to process the target first, observers may be compelled to initially allocate attention to unique locations. Yantis (1993) has shown that it is sometimes impossible to ignore display locations containing a unique feature value (i.e., a singleton) on a task-relevant dimension. Theeuwes (1991, 1992) and Hillstrom and Yantis (1994) have provided evidence that the unique element need not differ on a task-relevant dimension in order to capture attention, perhaps because unique elements often signal a novel percept.

\section{EXPERIMENT 3 Identification of Surround Orientation}

The results of Experiments 1 and 2 are consistent with the assertion that a featurally unique region attracts attention. In consequence, observers who are required to discern orientation of the texture target, as in Experiment 2, need not redirect attention when the detection process is completed. In the surround identification task of Experiment 1, however, attention must be released from the target region and reallocated to the surround before identification can proceed. This reorienting of attention is difficult to complete within the exposure duration of the display. A question that remains is whether the attentional capture associated with the target is a bottom-up (i.e., stimulus-driven) or a top-down (i.e., goal-directed) process. If it is a bottom-up process, then the effect is expected even when unique display regions are to be ignored (Yantis, 1993).

In Experiment 3, observers were instructed to report only the orientation of the surround and to ignore target presence and orientation. If the poor surround orientation accuracy found in Experiment 1 was due to the instruc- 
tions to attend first to the target or due to the dividedattention demands of the task, then surround orientation accuracy in Experiment 3 should equal the target orientation accuracy levels found in Experiment 2 and exceed surround orientation accuracy of Experiment 1. In addition, there should be no difference in surround orientation accuracy on target-present and target-absent trials. Alternatively, if attention is guided in a bottom-up manner by the signal from a GDO, then surround orientation accuracy should still be lower than target orientation accuracy (Experiment 2), and this should be particularly pronounced on target-present trials.

\section{Method}

Experiment 3 was conducted after the observers who participated in Experiments 1 and 2 were retested in Experiment 1. They were required only to identify the orientation of the surround, ignoring the target. All other stimuli and procedural details were identical to those reported in Experiment 1.

\section{Results}

Table 1 displays average orientation accuracy collapsed across trial type, separately for target-present and target-absent trials. Generally, accuracy was quite high $(93.44 \%)$ but was significantly worse on target-present trials $(89.96 \%)$ than on target-absent trials $(96.88 \%)$ $[t(4)=2.82, p<.05]$. Thus, the identification of surround orientation was better in the absence of a generalized difference.

We also compared surround orientation accuracy (Experiment 3), separately for target-present and target-absent trials, with target orientation accuracy (Experiment 2). In neither case was the difference significant $(p>.05)$.

\section{Discussion}

The results of Experiment 3 indicate that processing of surround features is more difficult when displays contain a target, even when observers are instructed to ignore target features. The performance decrements associated with target presence are consistent with the view that visual attention is captured by, and processing resources are allocated to, featurally unique regions of space (Cave \& Wolfe, 1990; Hillstrom \& Yantis, 1994; Theeuwes, 1991, 1992; Wolfe, 1994; Yantis, 1993). This tends to occur even when instructions favor a top-down, "feature search" strategy (Bacon \& Egeth, 1994; Yantis, 1993 ) and suggests that attentional capture by the texture patch is largely a bottom-up phenomenon.

\section{EXPERIMENT 4 Surround Orientation and Target Detection/Orientation}

Under the assumption that attention is captured by the target, one would expect target features to be processed better than surround features. While that has been the general trend in the present experiments, it is somewhat surprising that target orientation accuracy in Experiment 2 was not significantly better than surround orien- tation accuracy in Experiment 3, especially on targetpresent trials. It should be noted that this comparison would have been marginally significant under a onetailed test and that the power to detect better target performance was reduced because practice effects favored the surround orientation task. Experiment 4 was conducted to determine if superiority of target processing would be observed when practice effects were controlled.

\section{Method}

In this experiment, 4 undergraduates ( $M=24.8$ years of age, range $=22-26$ years) at the University of Calgary with no experience in laboratory texture segmentation tasks were tested using the procedures of Experiments 2 and 3 . They were asked to detect and identify the orientation of targets or to identify the orientation of the surrounding region while ignoring other display regions. Task order was counterbalanced to cont rol for practice effects. The observers were in good health, with normal or corrected visual acuity of 20/22 or better. Each observer was paid $\$ 5$ (Canadian) for participation in the single session, which lasted approximately $45 \mathrm{~min}$.

\section{Results}

Table 1 displays average accuracy for both target and surround tasks. When required only to identify the orientation of the surround, the effect of target presence was similar to, but larger than, that found with experienced observers in Experiment 3. The observers were most accurate when the target was absent $(92.93 \%)$, and they performed significantly worse when the target was present $(75.55 \%)[t(4)=6.59, p<.01]$. They had little difficulty at either detecting targets $(95.55 \%)$ or identifying their orientation on target-present trials $(92.98 \%)$. The difference between target detection and identification was nonsignificant $[t(4)=1.27, p>.05]$.

As in Experiment 3, we compared surround orientation accuracy on target-present trials with target orientation accuracy and found better performance for targets $[t(4)=3.61, p<.05]$. The target superiority effect was not observed relative to surround orientation accuracy on target-absent trials $[t(4)=0.02, p>.05]$.

\section{Discussion}

The results of Experiment 4 replicate in large part the findings of Experiments 2 and 3. The observers processed target features more accurately than they processed surround features. Target presence interfered with the processing of surround features even when the observers were instructed to attend only to the latter. Again, it appears that attention is directed quickly, in a bottom-up manner, to spatial locations possessing unique feature values.

This explanation is predicated on the hypothesis that the presence of a unique region is detected before specific features are identified. The results of Experiments 1,3 , and 4 are consistent with this view. Problematic are the data of Experiments 2 and 4, in which equivalent target detection and identification accuracy was obtained. However, the interpretation of these data is made difficult because performance was often at ceil- 
ing. To eliminate these ceiling effects and provide a direct test of the hypothesis, a comparison of target detection and identification as a function of exposure duration was required.

\section{EXPERIMENT 5 \\ Target Detection and Orientation Identification as a Function of Exposure Duration}

Sagi and Julesz (1985a, 1985b) and Atkinson and Braddick (1989) have suggested that the output of a GDO becomes available prior to featural details, allowing for detection and coarse localization prior to identification. Because texture segmentation is believed to be based on the output of a GDO (Nothdurft, 1985b, 1991a, 1991b, 1992), detection of the texture patch should precede the identification of features within the patch. To test this hypothesis, a new group of observers was required to detect a texture target and then report its orientation. At relatively long exposure durations, both detection and identification should be near perfect, as in Experiment 2. The shorter durations are of greatest interest, because, at these durations, detection should be superior to identification accuracy.

\section{Method}

Observers. Four experimentally naive undergraduates $(M=27$ years of age, range $=24-32$ years) at the University of Calgary participated. All had normal or corrected visual acuity of $20 / 20$ or better. Each observer was paid \$10 (Canadian) for participating in a testing session, which lasted approximately $1 \mathrm{~h}$.

Stimuli. With one exception, display features were identical to those used in Experiment 2, in which surround items were always oriented vertically and target items oriented $45^{\circ}$ to the left or right of vertical. Unlike in Experiment 2, targets were not presented at fixation.

Procedure. The observers were required to first detect target presence and then report its orientation in five conditions that differed in the duration of texture displays: 14, 21, 28, 49, and $70 \mathrm{msec}$. Within each condition, two blocks of 32 trials each were presented. All other procedural details were identical to those used in Experiment 2. Duration conditions were presented in counterbalanced order.

\section{Results}

As shown in Figure 2, target detection proved to be the easier task overall. In addition, both target detection and orientation identification accuracy improved with exposure duration. However, at exposure durations ranging from 14 to $28 \mathrm{msec}$, target detection accuracy was higher than identification accuracy; at exposure durations of 49 and $70 \mathrm{msec}$, both were approximately equal.

The relationships among target detection, orientation identification, and display duration were analyzed in a task (2) $\times$ time (5) univariate mixed-model analysis of variance (ANOVA). A Geisser-Greenhouse correction (see Maxwell \& Delaney, 1990) was used because the data violated the sphericity (circularity) assumption of the traditional, mixed-model ANOVA. A data set possesses sphericity if each relevant difference score has the same variance.

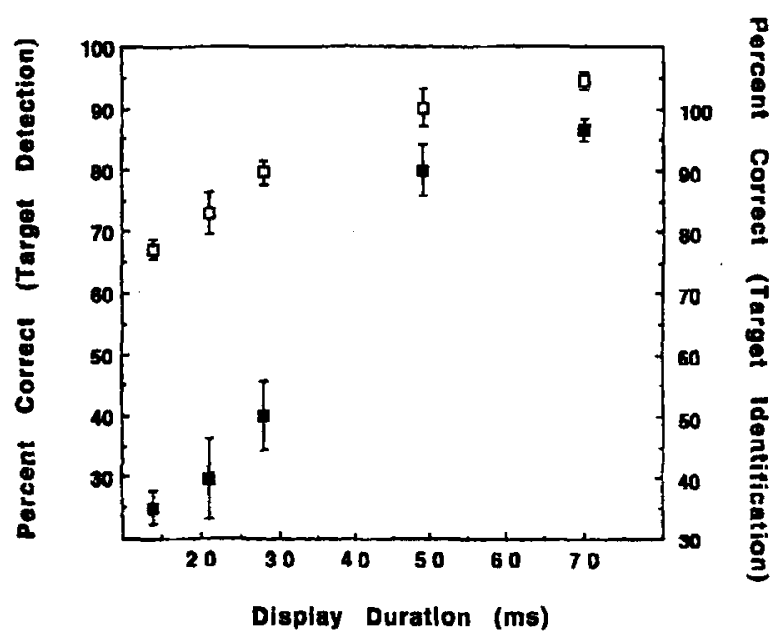

Figure 2. Target detection (open symbols) and orientation identification accuracy (filled symbols) as a function of exposure duration for Experiment 5. Note the different $y$-axes for detection and orientation identification. $S E$ bars are included.

The main effects of task $[F(1,3)=144.03, p<.01]$ and time $[F(2.01,6.04)=121.15, p<.01]$ reached significance, as did their interaction $[F(1.94,5.83)=25.35$, $p<.01]$. The linear trend to the task $\times$ time interaction was significant $(p<.01)$, indicating that the differences between detection and identification accuracy decreased as a linear function of exposure duration.

\section{Discussion}

The results of Experiment 5 support the view that the output of the GDO is available prior to information regarding specific target features. As the output of a GDO becomes available, detection and coarse localization can occur, whereas fine localization and identification must await the output of a slower feature comparison process (Atkinson \& Braddick, 1989; Sagi \& Julesz, 1985a, 1985b). This may explain why feature search targets can be detected but not well localized (Treisman \& Gelade, 1980). To be sure, Duncan (1993, Experiment 2) suggested that localization and identification can occur simultaneously in a single object, but his design did not allow for a comparison of the time courses of these processes.

\section{GENERAL DISCUSSION}

The high accuracy levels found for target detection in Experiments 1, 2, and 4 are consistent with the view that, with a sufficient gradient, texture segmentation is automatic, requiring little in the way of higher level processing. Target orientation accuracy was also near perfect in both practiced and unpracticed, naive observers. Generally, results indicated that surround orientation accuracy was significantly worse than target orientation accuracy, especially on target-present trials. 
Why is it more difficult to report the feature values corresponding to nontarget regions? Several explanations of the target superiority effect have been ruled out. Differential practice cannot account for the effect, which was still found in the naive observers in Experiment 4 . It was not due to interference produced by responding to target features. Even when they were told to ignore the target and report only surround orientation (Experiment 4), instructions that should have led them to adopt a feature search strategy (Bacon \& Egeth, 1994), they were less accurate than when asked to report target orientation. In addition, the results of Experiments 3 and 4 indicated poorer identification of surround features when the target was present than when the target was absent. This too suggests that a featurally unique region captures visual attention.

The results of Experiment 5 allow one to argue that attentional capture by a unique region occurs because the output of a GDO guides attention to a local discontinuity before specific features have been identified. When the region containing the discontinuity is also the region containing to-be-identified features, facilitation is the result. However, when the features of other regions must be identified, attention must be released from the area corresponding to the discontinuity.

This view is not terribly different from GS2 (Wolfe, 1994), in which a categorical GDO and feature-comparison stage are used to guide attention during texture segmentation and search. The preceding experiments suggest a revision to GS2-namely, that attention will be guided continuously by whatever information is available and that the information available first is often related to local differences.

Hillstrom and Yantis (1994), Yantis (1993), and Bacon and Egeth (1994) have argued that goal-directed allocation of attention underlies most visual search involving singleton detection. Feature s arch may well be influenced by myriad top-down process - s, but we would argue that the texture segmentation data presented here, and in particular the target superiority effect, are largely bottom-up phenomena. The strongest evidence can be found in Experiment 4, where naive observers were instructed specifically to ignore the target and to discern surround orientation. It is clear that the presence of a local difference interfered with processing; surround orientation accuracy was significantly reduced on targetpresent trials. Thus, even when observers are instructed to identify specific features, irrespective of a local difference, this difference still attracts visual attention. It seems, then, that featurally unique regions are given greater priority.

Practice resulted in considerable improvement in the identification of features composing nontarget regions. It is possible that familiarity with the displays and task allows observers to more quickly detect targets and reallocate attention to surrounding regions. It is also possible that they become better at ignoring information provided by the GDO (see Wolfe, 1994) and rely increasingly on the feature comparison stage. Even if they were able to utilize information based on feature comparisons, the evidence suggests that they were unable to completely escape the influence of the GDO. Surround identification accuracy was always higher on target-absent trials than on target-present trials. One might be tempted to argue that this occurred because it is harder to identify a region's orientation when two orientations are present. This argument can be dispelled, however, because it would predict that identification of target orientation should be worse than surround orientation identification on target-absent trials.

A prediction derived from this account is that access to nontarget features should be good when they are neither more nor less unique than targets. In this case, the output of the GDO does not guide attention to a specific region. This prediction can be tested by presenting observers with a spatial two-alternative forced-choice task in which the target and nontargets each occupy $50 \%$ of the display. Both targets and nontargets would be allowed to take on two feature values (e.g., vertical or horizontal targets and $45^{\circ}$ oblique nontargets). Under these conditions, observers should be equally accurate at reporting target and nontarget orientation.

\section{REFERENCES}

AtKinson, J., \& BradDick, O. J. (1989). "Where" and "what" in visual search. Perception, 18, 181-189.

BACON, W. F., \& EGETH, H. E. (1994). Overriding stimulus-driven attentional capture. Perception \& Psychophysics, 55, 485-496

CAVE, K. R., \& WolfE, J. M. (1990). Modeling the role of parallel processing in visual search. Cognitive Psychology, 22, 225-271.

DunCAN, J. (1984). Selective attention and the organization of visual information. Journal of Experimental Psychology: General, 113, 501-517.

DUNCAN, J. (1993). Coordination of what and where in visual attention. Perception, 22, 1261-1270.

Findlay, J. M. (1982). Global visual processing for saccadic eye movements. Vision Research, 22, 1033-1045.

Hillstrom, A. P., \& Yan'ts, S. (1994). Visual motion and attentional capture. Perception \& Psychophysics, 55, 399-4I1.

JONIDES, J., \& YANTIS, S. (1988). Uniqueness of abrupt visual onset in capturing attention. Perception \& Psychophysics, 43, 346-354.

JulEsZ, B. (1986). Texton gradients: The texton theory revisited. Biological Cybernetics, 54, 245-251.

LANDY, M., \& BERGEN, J. (1991). Texture segregation and orientation gradient. Vision Research, 31, 679-691.

Malik, J., \& Perona, P. (1990). Preattentive texture discrimination with early vision mechanisms. Journal of the Optical Society of America, 7, 923-932.

Maxwell, S. E., \& Delaney, H. D. (1990). Designing experiments and analyzing data: A model comparison perspective. Belmont, $\mathrm{CA}$ : Wadsworth.

Nothdurft, H. C. (1985a). Orientation sensitivity and texture segmentation in patterns with different line orientation. Vision Research, 25, 551-560.

Nothdurf, H. C. (1985b). Sensitivity for structure gradient in texture discrimination tasks. Vision Research, 25, 1957-1968.

NothduRfT, H. C. (1991a). Different effects from spatial frequency 
masking in texture segregation and texton detection tasks. Vision Research, 31, 299-320.

Nothdurf,, H. C. (1991b). Texture segmentation and pop-out from orientation contrast. Vision Research, 31, 1073-1078.

Nothdurft, H. C. (1992). Feature analysis and the role of similarity in preattentive vision. Perception \& Psychophysics, 52, 355-375.

Ottes, F. P., Van Gisbergen, J. A. M., \& Eggermont, J. J. (1984). Metrics of saccade responses to visual double stimuli: Two different modes. Vision Research, 24, 1169-1179.

Ottes, F. P., Van Gisbergen, J. A. M., \& Eggermont, J. J. (1985). Latency dependence of colour-based target vs. nontarget discrimination by the saccadic system. Vision Research, 25, 849-862.

RENSinK, R. A., \& EnNs, J. T. (1993). VScope Version 1. $2 b$ [Computer program]. University of British Columbia.

SAGI, D., \& Julesz, B. (1985a). Detection versus discrimination of visual orientation. Perception, 14,619-628.

SAGI, D., \& Julesz, B. (1985b, June). "Where" and "what" in vision. Science, 228, 187-198.

SAGI, D, \& JuLESZ, B. (1987). Short-range limitation on detection of feature differences. Spatial Vision, 2, 39-49.
Theeuwes, J. (1991). Cross-dimensional perceptual selectivity. Perception \& Psychophysics, 50, 184-193.

TheEuwes, J. (1992). Perceptual selectivity for color and form. Perception \& Psychophysics, 51, 599-606.

Treisman, A. M., \& Gelade, G. (1980). A feature-integration theory of attention. Cognitive Psychology, 12, 97-136.

VoorheEs, H., \& Poggio, T. (1988). Computing texture boundaries from images. Nature, 333, 364-367.

WOLFE, J. M. (1992). "Effortless" texture segmentation and "parallel" visual search are not the same thing. Vision Research, 32, 757-763.

WOLFE, J. M. (1994). Guided Search 2.0: A revised model of visual search. Psychonomic Bulletin \& Review, 1, 202-238.

YANTIS, S. (1993). Stimulus-driven attentional capture. Current Directions in Psychological Science, 2, 156-161.

(Manuscript received December 16, 1994; revision accepted for publication May 2, 1995.) 